\title{
Biochemical Studies of a Patient with Hereditary Hepatorenal Tyrosinemia: Evidence of Glutathione Deficiency
}

\author{
ELIZABETH STONER, HAROLD STARKMAN, DANIEL WELLNER, VAIRA P. WELLNER, \\ SHIGERU SASSA, ARLEEN B. RIFKIND, ANDRE GRENIER, PETER G. STEINHERZ, \\ ALTON MEISTER, MARIA I. NEW, AND LENORE S. LEVINE
}

Department of Pediatrics, Division of Pediatric Endocrinology [E.S., H.S., M.I.N., L.S.L.], Department of Biochemistry [D.W., V.P.W., A.M.], and Department of Pharmacology and Medicine [A.B.R.], The New York Hospital-Cornell Medical Center, New York, New York 10021, The Rockefeller University, New York, New York 10021 [S.S.], Department of Human Genetics, Le Centre Hospitalier de l'Universite Laval, Quebec, Canada [A.G.], and Memorial Sloan-Kettering Cancer Center, New York, New York 10021 [P.G.S.]

\begin{abstract}
Metabolic and enzymatic studies in a patient with hereditary tyrosinemia demonstrated for the first time a deficiency of erythrocyte and hepatic glutathione. Markedly decreased hepatic fumarylacetoacetate hydrolase activity was demonstrated in this patient. The activities of hepatic enzymes not involved in tyrosine metabolism were also determined. Assay of mixed function oxidase activity demonstrated low levels of aryl hydrocarbon hydroxylase and 7-ethoxycoumarin deethylase, suggesting decreased hepatic detoxification capacity. 5-Aminolevulinic acid dehydratase activity was undetectable. Succinylacetone (4,6dioxoheptanoic acid), an abnormal metabolic product secondary to fumarylacetoacetate hydrolase deficiency was found in serum and urine. Succinylacetone was demonstrated to inhibit 5-aminolevulinic acid dehydratase in vitro, as did the urine, plasma, and red cell lysates of the patient. (Pediatr Res 18:1332-1336, 1984)
\end{abstract}

Hereditary hepatorenal tyrosinemia is a rare metabolic disorder characterized by increased levels of tyrosine in the blood and urine. It presents during early childhood with hepatocellular disease, Fanconi's syndrome, and hypophosphatemic rickets. A pathognomonic feature of this disease is the presence of succinylacetone (4,6-dioxoheptanoic acid) in serum and urine (18). Malignant hepatoma, prevalent in this disorder, is not explained solely by cirrhosis (35).

The primary enzymatic defect in tyrosinemia is not known. Recently it has been postulated to be a deficiency of fumarylacetoacetate hydrolase $(2,18)$. Several additional enzymes have also been found to be deficient, as in this report and previous publications. Many clinical and biochemical findings remain unexplained in tyrosinemia. The aim of our study was to increase our understanding of this disease by examining the biochemical abnormalities and hepatotoxicity in such a patient. Our investi-

Received October 3, 1983; accepted March 13, 1984.

Correspondence may be addressed to Elizabeth Stoner, M.D., Division of Pediatric Endocrinology, The New York Hospital-Cornell Medical Center, 525 East 68th Street, New York, NY 10021.

This investigation was supported in part by Grants AM-06354, AM-12034, HD00072 , and ES-02165 from the National Institutes of Health, and was also supported by Grant RR 47 from the National Institutes of Health, Division of Research Resources, General Clinical Research Centers Program. gation revealed hepatic and erythrocyte glutathione deficiency, and decreased activity of several hepatic enzymes: fumarylacetoacetate hydrolase, 5-aminolevulinic acid dehydratase, and mixed function oxidases. The deficiencies of glutathione and mixed function oxidases, which have not been previously reported in tyrosinemia, may play a significant role in the carcinogenic potential associated with this disorder.

\section{CASE HISTORY}

The patient was a $1 \frac{1}{2}-$-year-old female admitted to the Pediatric Clinical Research Center at The New York Hospital-Cornell Medical Center. Pregnancy and neonatal course were uncomplicated. There was no jaundice. The baby was breastfed for the first $10 \frac{1}{2}$ months. Although she fed well, she was irritable, and vomited daily. At the time of the evaluation she was on a diet appropriate for her age. Developmental 'milestones occurred normally until 6 months of age, but she was not standing or making sounds at 13 months.

At 9 months of age, hemoglobin was $12.5 \mathrm{~g} / \mathrm{dl}$. By 1 year, it had decreased to $10.5 \mathrm{~g} / \mathrm{dl}$, and the patient had a palpable spleen.

Physical examination at 14 months of age revealed a weight of $9.34 \mathrm{~kg}$ (25th percentile), height of $79.3 \mathrm{~cm}$ (50th percentile), and head circumference of $44.5 \mathrm{~cm}$ (15th percentile). Abnormal physical findings included a grade II/VI systolic murmur at the apex without radiation, an enlarged liver $(10.5-\mathrm{cm}$ span at the midclavicular line), and the tip of the spleen was palpable $2 \mathrm{~cm}$ below the left costal margin. Neurologic examination revealed poor muscle strength proximally in the lower extremities, but was otherwise within normal limits.

Plasma tyrosine, methionine, bile acids, and ammonia were elevated (Table 1). Renal tubular dysfunction with acidosis was noted. $\alpha$-Fetoprotein was markedly elevated. Serum cholesterol and triglycerides were also elevated, associated with fasting hypoglycemia and undetectable serum insulin. There was no response to glucagon in the fasting state or postprandially. Galactose-1-phosphate uridyltransferase was within normal limits. Serum calcium was normal, with a markedly decreased serum phosphorus. Alkaline phosphatase, primarily of bone origin, was elevated. Parathyroid hormone and vitamin $D$ levels were normal. Bone age was appropriate for age. Early signs of rickets in the wrists and knees were demonstrated radiographically. An abdominal sonogram showed hepatosplenomegaly with no signs of increased portal pressure or tumor. An electroencephalogram 
Table 1. Biochemical results in patient with hereditary hepatorenal tyrosinemia

\begin{tabular}{|c|c|}
\hline Metabolic & Hepatic \\
\hline $\begin{array}{l}\text { Plasma amino acids* } \\
\quad \text { Tyrosine, } 751 \mu \mathrm{M}(21-87 \mu \mathrm{M}) \\
\text { Methionine, } 989 \mu \mathrm{M}(6-39 \mu \mathrm{M}) \\
\text { Urinary amino acids: increased threonine, serine, } \\
\quad \text { glycine, alanine, methionine, isoleucine, tyrosine } \\
\text { Cholesterol, } 206 \mathrm{mg} / \mathrm{dl} \text { l }\} \text { (>97th percentile for age) } \\
\text { Triglycerides, } 122 \mathrm{mg} / \mathrm{dl} \\
\text { Ferric chloride test, within normal limits } \\
\text { Berry spot test, negative } \\
\text { Urinary catechols, } 52 \mu \mathrm{g}(8-160 \mu \mathrm{g}) \\
\text { Urinary uroporphyrins, nondetectable (WNL) } \dagger \\
\text { Urinary porphobilinogen, nondetectable (WNL) } \\
\text { Thyroid function tests, within normal limits }\end{array}$ & 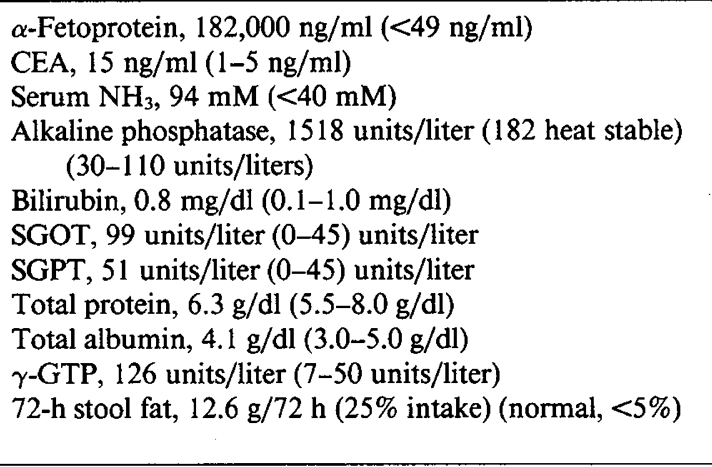 \\
\hline Renal & Hematologic \\
\hline
\end{tabular}

* Normal values are in parentheses.

$\dagger$ WNL, within normal limits; CEA, carcinoembryonic antigen; SGOT, serum glutamate oxaloacetate transaminase; SGPT, serum glutamate pyruvate transaminase, $\gamma$-GTP, $\gamma$-glutamyl transpeptidase.

showed mild and nonspecific changes characterized by rare sharp wave activity. Computerized transaxial tomography of the brain demonstrated mild cerebral atrophy.

Liver biopsy showed cirrhosis with nodular areas and fat deposition on gross examination. Light microscopy revealed focal regenerative nodules with a variable degree of fibrous tissue. Individual nodules showed variable amounts of steatosis, cholestasis, and numerous acidophilic bodies. All evaluations were performed under a protocol approved by this institution's review boards. Informed consent was obtained from the parents.

\section{MATERIALS AND METHODS}

Hepatic enzymes of tyrosine metabolism. Tyrosine aminotransferase and 4-hydroxyphenylpyruvate dioxygenase activities were measured according to the method of Whelan and Zannoni (36), and maleylacetoacetate isomerase and fumarylacetoacetate hydrolase activities were measured by the methods of Edwards and Knox (4).

Pyrrole metabolism. Porphyrins, 5-aminolevulinic acid dehydratase, and uroporphyrinogen synthetase were measured using methods previously reported $(29,30)$. A portion of the liver obtained at biopsy was homogenized in $0.1 \mathrm{M}$ sodium phosphate buffer, $\mathrm{pH} 7.4$, and the homogenate was then centrifuged at 9000 $\times g$ for $10 \mathrm{~min}$. The porphyrin content of the liver (9) and the activity of 5-aminolevulinic acid dehydratase (31) was measured in the homogenate. The activity of 5-aminolevulinic acid synthase was measured in the $9000 \times g$ pellet (31).

Glutathione determination. A portion of the liver biopsy specimen was sonicated for $30 \mathrm{~s}$ in 5 volumes of $1 \%$ picric acid, centrifuged, and glutathione concentration was measured using the method of Tietze (33). Erythrocyte glutathione was measured by the same method immediately after hemolyzing the cells by dilution with 100 volumes of water.

Mixed function oxidases. The activities of aryl hydrocarbon hydroxylase and 7-ethoxycoumarin deethylase were measured in the $9000 \times g$ supernatant $(26)$.

\section{RESULTS}

Activity of enzymes of tyrosine metabolism measured in liver tissue are presented in Table 2. These studies indicated the absence of 4-hydroxyphenylpyruvate dioxygenase activity, markedly decreased fumarylacetoacetate hydrolase activity, decreased tyrosine aminotransferase activity as well as normal maleylacetoacetate isomerase and increased homogentisate dioxygenase activity.

Liver and erythrocyte glutathione concentrations were significantly decreased (Table 3 ).

Serum and urinary determination of organic acids by mass spectrometry showed the presence of succinylacetone and 4hydroxybenzaldehyde, as well as markedly elevated levels of lactate and 4-hydroxyphenyllactate (39).

The activities of enzymes and intermediates involved in heme biosynthesis and microsomal hemoprotein function are presented in Table 4. In erythrocytes, 5-aminolevulinic acid dehydratase was undetectable, whereas uroporphyrinogen-I synthetase was at the upper limits of normal. Erythrocyte protoporphyrin content was slightly elevated, a finding consistent with new erythrocyte production in response to anemia. In liver, 5aminolevulinic acid dehydratase activity was also undetectable. Consistent with the marked inhibition of 5-aminolevulinic acid dehydratase activity, hepatic porphyrins were below normal (3). In contrast, the activity of 5-aminolevulinic acid synthase, the rate-limiting enzyme for heme synthesis in liver, was more than three times higher than values reported for normal adults, and was in the range observed in patients with porphyria $(12,22,34)$.

Activities of the mixed function oxidases, aryl hydrocarbon hydroxylase and 7-ethoxycoumarin deethylase (Table 4), were at the lower end of the range observed in human fetal liver $(25$, 27 ), and only $1-10 \%$ of the mean values reported for autopsy specimens of adult liver (11).

\section{DISCUSSION}

In this study, we have documented the extensive hepatic, renal, and skeletal abnormalities previously reported in tyrosinemia, 
and we report several additional biochemical derangements. The primary defect in this disease has been postulated to be a decreased activity of fumarylacetoacetate hydrolase (18). This enzyme, first reported in mammalian liver, kidney, and other tissues as having acylpyruvic acid-hydrolyzing activity (19), catalyzes the final step in the main phenylalanine and tyrosine degradative pathway (Fig. 1, Reaction 6). We found a low hepatic activity of this enzyme in our patient, confirming similar findings by others $(2,5,14,15)$. A deficiency of this enzyme is postulated to account for the presence of succinylacetone and succinylacetoacetate in the urine of patients with tyrosinemia (18). Succinylacetoacetate presumably arises by reduction of either maleylacetoacetate or fumarylaceotacetate or both. The mechanism of its formation is unknown. Its absence in the urine of normal individuals may be attributable to the fact that it is a substrate of the hydrolase (13). Neither fumarylacetoacetate nor maleylacetoacetate has been found in the urine of patients with tyrosinemia.

Hypoglycemia and acidosis were prominent findings in this patient (Table 1). The failure of lactate to increase with concomitant hypoglycemia after glucagon stimulation differentiates this disorder from glycogen storage diseases. The lack of response to glucagon may be secondary to cirrhosis and a resultant decrease in total liver glycogen. Alternatively, the enzymes necessary for glycolysis may be inhibited by abnormal metabolic products.

Table 2. Hepatic enzymes of tyrosine metabolism*

\begin{tabular}{lcc}
\hline \multicolumn{1}{c}{ Enzyme } & \multicolumn{2}{c}{$\begin{array}{c}\text { Range of normal } \\
\text { controls }(n=10)\end{array}$} \\
\hline Tyrosine aminotransferase & 0.024 & $0.027-0.091$ \\
4-Hydroxyphenylpyruvate dioxygenase & $<0.004$ & $0.085-0.144$ \\
Homogentisate dioxygenase & 1.50 & $0.65-1.24$ \\
Maleylacetoacetate isomerase & 0.77 & $0.31-2.21$ \\
Fumarylacetoacetate hydrolase & 0.15 & $0.60-1.17$ \\
\hline
\end{tabular}

*Values are expressed as units/mg protein ( 1 unit $=1 \mu \mathrm{mol}$ of substrate metabolized/60 $\mathrm{min}$ at $22^{\circ} \mathrm{C}$, except for homogentisate dioxygenase which was measured at $37^{\circ} \mathrm{C}$ ).

Table 3. Hepatic and erythrocyte glutathione

\begin{tabular}{lcc}
\hline Hepatic glutathione $(\mu \mathrm{mol} / \mathrm{g}$ tissue $)$ & \\
Patient & & 3.4 \\
Normal control liver biopsy & & 5.9 \\
Erythrocyte glutathione & $\mu \mathrm{mol} / \mathrm{ml}$ of blood & $\frac{\mathrm{nmol} / \mathrm{mg} \mathrm{Hb}}{2.9^{*}}$ \\
Patient & $0.35^{*}$ & 4.7 \\
Father & 0.84 & 6.8 \\
Mother & 1.32 & $6.1 \dagger$ \\
Controls & $0.92 \dagger$ & \\
\hline
\end{tabular}

* Mean of four determinations over 4 months (range, $0.26-0.46 \mu \mathrm{mol} /$ / $\mathrm{ml}$ of blood; $2.3-3.6 \mathrm{nmol} / \mathrm{mg} \mathrm{Hb}$ ).

$\dagger$ Mean of five samples (range, $0.74-1.12 \mu \mathrm{mol} / \mathrm{ml}$ of blood; $4.0-8.7$ $\mathrm{nmol} / \mathrm{mg} \mathrm{Hb}$ ).
Impaired gluconeogenesis and glycogenolysis induces lipolysis, causing the increase in serum cholesterol and triglycerides noted. The delay in developmental milestones and cerebral cortical atrophy may be the result of prolonged episodes of hypoglycemia or may be secondary to the toxic effects of the metabolic derangement of amino acid metabolism.

The increased level of tyrosine in the blood has been attributed

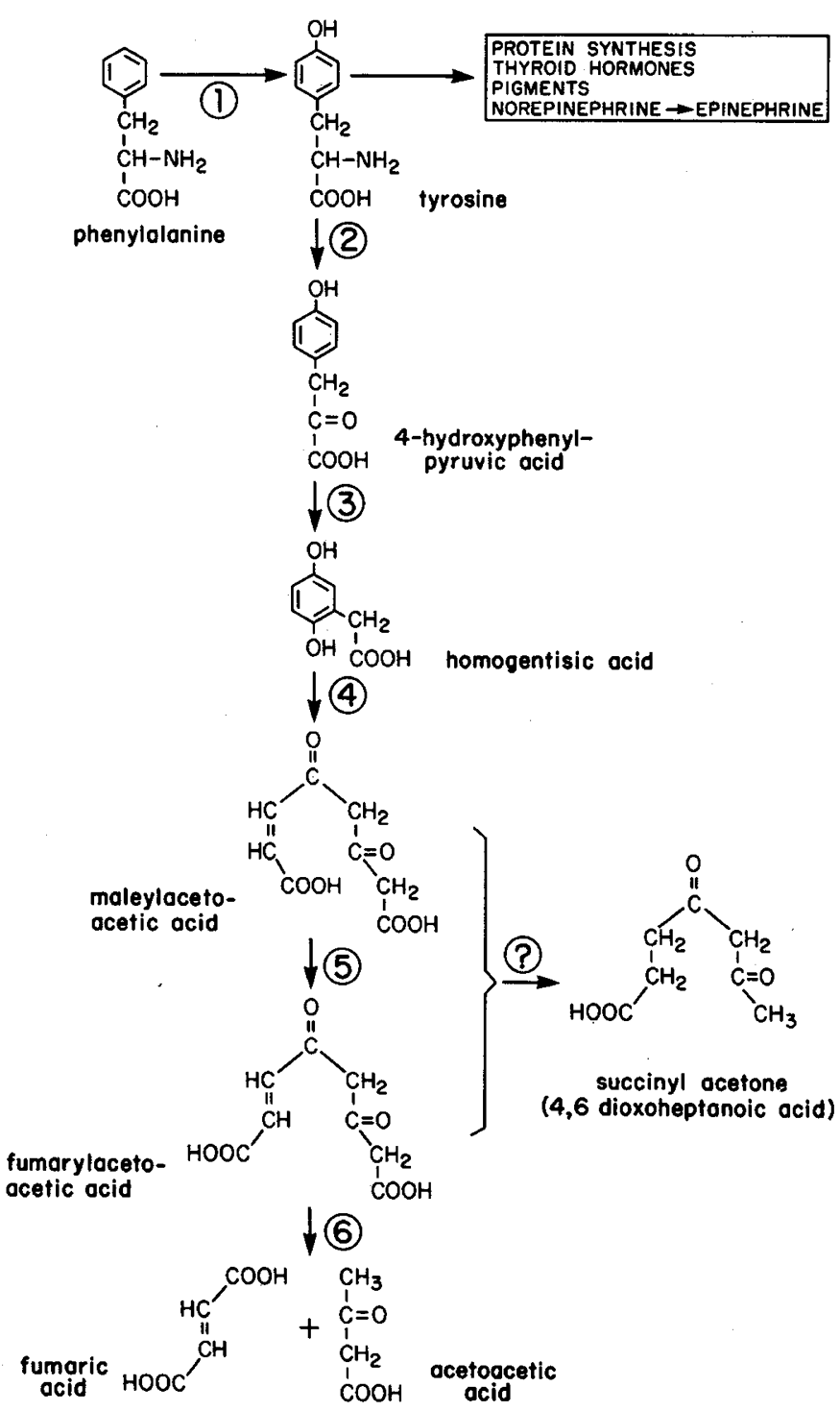

Fig. 1. Tyrosine degradative pathway: intermediary metabolism. 1 , phenylalanine hydroxylase; 2, tyrosine aminotransferase; 3, 4-hydroxyphenylpyruvate dioxygenase; 4 , homogentisate dioxygenase; 5 , maleylacetoacetate isomerase; 6 , fumarylacetoacetate hydrolase.

Table 4. Enzymes and intermediates involved in heme biosynthesis and function*

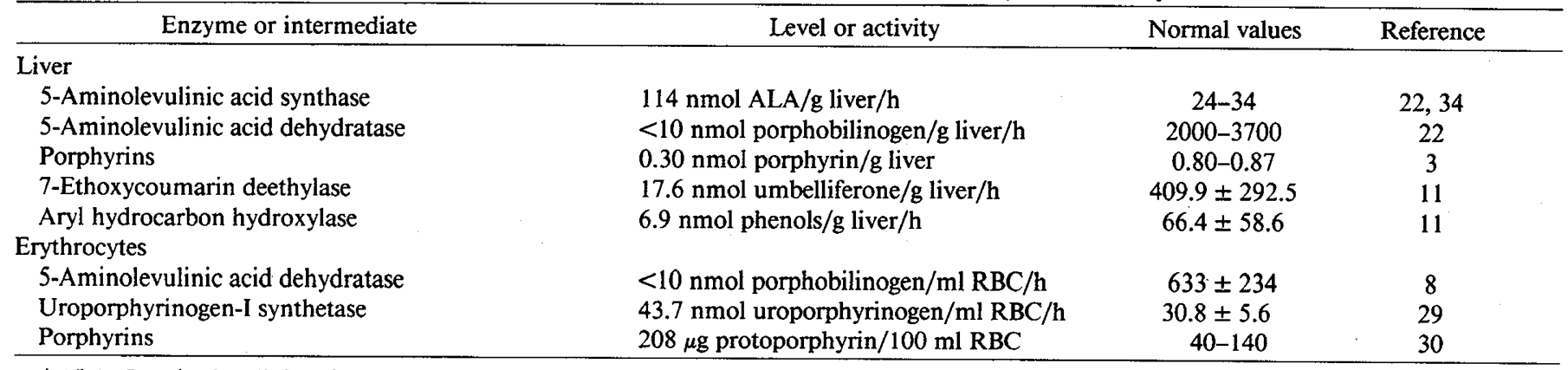

*ALA, 5-aminolevulinic acid; RBC, red blood cells. 
to low hepatic 4-hydroxyphenylpyruvate dioxygenase activity $(7$, 16, 17), (Fig. 1, Reaction 3). The activity of this enzyme may also be decreased by the toxic metabolites produced in this condition. The increased methionine level, a variable finding in this disorder (20), has been explained by secondary inhibition of methionine-activating enzyme and cystathionine synthase (6).

Several abnormalities of heme metabolism have been reported in tyrosinemia. These include increased urinary excretion of 5 aminolevulinic acid $(6,7,10)$, decreased activity of liver and erythrocyte 5-aminolevulinic acid dehydratase $(18,32)$, increased activity of 5 -aminolevulinic acid synthetase $(7,10)$, and porphyria-like symptoms.

In our patient, 5-aminolevulinic acid dehydratase activity was undetectable in the liver, less than $1 \%$ of normal in erythrocytes, and was not restored, even after transfusion and red cell washing. Both parents of the child had normal erythrocyte levels of 5aminolevulinic acid dehydratase activity. The activity of 5-aminolevulinic acid synthase was elevated in the liver of the patient, and there was high urinary excretion of 5-aminolevulinic acid. Lindblad et al. (18) showed that succinylacetone directly inhibits 5-aminolevulinic acid dehydratase, and postulated that the increase in 5-aminolevulinic acid excretion observed in tyrosinemia is secondary to inhibition by succinylacetone.

The low levels of mixed function oxidase activity observed in this patient have not been described previously in tyrosinemia. Levels could be low as a consequence of impaired heme percursor and cytochrome $P-450$ synthesis, structural liver damage accompanying cirrhosis, direct inhibition of mixed function oxidase activity by succinylacetone, or other organic acids, or a combination of these factors. The low levels of mixed function oxidase activity could result in a decreased detoxification capacity of the liver and may contribute to the development of hepatic cirrhosis and hepatocellular carcinoma.

This is the first report of a depression of glutathione in erythrocytes and liver of a patient with tyrosinemia. Fumarylacetoacetate has been shown to react with glutathione to form an adduct (4). If fumarylacetoacetate accumulates in tyrosinemia as suggested, this reaction may explain the glutathione depletion we observe. Glutathione forms an adduct with maleylacetoacetate much more slowly or not at all. However, glutathione both catalyzes the isomerization of maleylacetoacetate to fumarylacetoacetate, with which it can then react, and also serves as a coenzyme for the isomerase (4). Thus, it is possible that the isomerase activity may be deficient in tyrosinemia because of the glutathione depletion. Another possible reason for the low glutathione concentrations is that its synthesis may be inhibited by some of the organic acids which accumulate in tyrosinemia.

Glutathione is known to act as a scavenger for a large number of toxic metabolites (1). It also serves to protect the integrity of cell membranes and to maintain protein sulfhydryl groups in the reduced state. Thus, low levels of glutathione may contribute to liver toxicity and malignant potential in tyrosinemia by impairing the detoxification of toxic metabolites.

Treatment with glutathione or other thiols may thus offer a means of decreasing the level of toxic and carcinogenic compounds in patients with tyrosinemia. Glutathione and cysteine have been shown to protect against chemically induced toxicity (21) and mutagenesis (28). Administration of glutathione has been reported to induce regression of liver tumors in animals (23). Treatment of acetaminophen toxicity with cysteamine or $N$-acetylcysteine, a glutathione precursor, has proven successful (24). L-2-Oxothiazolidine-4-carboxylate has been shown to be an effective glutathione precursor in experimental animals $(37,38)$ and may be a valuable potential therapeutic agent in tyrosinemia.

In summary, metabolic studies have demonstrated extensive enzymatic and biochemical abnormalities in a patient with tyrosinemia. We have demonstrated several enzyme abnormalities not previously described. The decreased glutathione in liver and erythrocytes which we report for the first time may play an important role in the pathophysiology of this disease. The de- creased levels of glutathione demonstrated in our patient suggest the possible benefit of a new mode of therapy, aimed at increasing the concentration or availability of glutathione in tissues, particularly in liver.

Acknowledgment. We wish to thank Dr. O. Mamer for the gas chromatography-mass spectrometry analysis of serum and urinary organic acids.

\section{REFERENCES}

1. Arias IM, Jakoby WB 1976 Glutathione: Metabolism and Function, Kroc Foundation Series, Vol 6. Raven Press, New York

2. Berger R, Smit GPA, Staker-de Vries SA, Duran M, Ketting D, Wadman SK 1981 Deficiency of fumarylacetoacetase in a patient with hereditary tyrosinemia. Clin Chim Acta 114:37

3. Doss M, Schmeruly E, Look D, Henning H, Hocevar V, Dohmen K, Anlauf M 1976 Studies on the metabolism of $\Delta$-aminolevulinic acid and porphobilinogen in liver biopsies from patients with acute intermittent porphyria. In: Jones J (ed) Porphyrins in Human Disease. Karger, Basel, pp 205-215

4. Edwards SW, Knox WE 1956 Homogentisate metabolism: the isomerization of maleylacetoacetate by an enzyme which requires glutathione. J Biol Chem 220:79

5. Fallstrom SP, Lindblad B, Lindstedt S, Steen G 1979 Hereditary tyrosinemiafumarylacetoacetase deficiency. Pediatr Res 13:78

6. Gaull GE, Rassin DK, Solomon GE, Harris RC, Sturman JA 1970 Biochemical observations in so-called hereditary tyrosinemia. Pediatr Res 4:337

7. Gentz J, Hohansson S, Lindblad B, Lindstedt S, Zetterstrom R 1969 Excretion of $\Delta$-aminolevulinic acid in hereditary tyrosinemia. Clin Chim Acta 23:257

8. Granick JL, Sassa S, Granick S, Levere RD, Kappas A 1973 Studies in lead poisoning. II. Correlation between the ratio of activated to inactivated $\Delta$ aminolevulinic acid dehydratase of whole blood and the blood lead level. Biochem Med 8:149

9. Granick S, Sinclair P, Sassa S, Grieninger G 1975 Effects by heme, insulin and serum albumin on heme and protein synthesis in chick embryo liver cells cultured in a chemically defined medium and a spectrofluorometric assay for porphyrin composition. J Biol Chem 250:9215

10. Kang ES, Gerald PS 1970 Hereditary tyrosinemia and abnormal pyrrole metabolism. J Pediatr 77:397

11. Kapitulnik J, Poppers PJ, Conney AH 1977 Comparative metabolism of benzo(a)pyrene and drugs in human liver. Clin Pharmacol Ther 21:166

12. Kaufman L Marver HS 1970 Biochemical defects in two types of human hepatic porphyria. N Engl J Med 283:954

13. Knox WE, Edwards SW 1955 The properties of maleylacetoacetate, the initial product of homogentisate oxidation in liver. J Biol Chem 216:489

14. Kvittingen EA, Halvorsen S, Jellum E 1983 Deficient fumarylacetoacetate fumarylhydrolase activity in lymphocytes and fibroblasts from patients with hereditary tyrosinemia. Pediatr Res 14:541

15. Kvittingen EA, Jellum E, Stokke D 1981 Assay of fumarylacetoacetate hydrolase in human liver-deficient activity in a case of hereditary tyrosinemia. Clin Chim Acta 115:311

16. LaDu BN, Gjessing LR 1978 Tyrosinosis and tyrosinemia. In: JB (ed) The Metabolic Basis of Inherited Disease, Stanbury, Chap 12. McGraw Hill, New York, p 256

17. Lindblad B, Lindstedt G, Lindstedt S, Rundgren M 1972 Metabolism of $p$ hydroxyphenylpyruvate in hereditary tyrosinemia. In: Stein J, Toohill C (eds) Organic Aciduria. Churchill Livingston, London, p 63

18. Lindblad B, Lindstedt S, Steen 1977 On the enzymatic defects in hereditary tyrosinemia. Proc Natl Acad Sci USA 74:4641

19. Meister A. Greenstein JP 1948 Enzymatic hydrolysis of 2,4-diketoacids. J Biol Chem 175:573

20. Michaels K, Matalon R, Wong PWK 1978 Dietary treatment of tyrosinemia type I. J Am Diet Assoc 73:507

21. Mitchell JR, Jollow DJ, Potter WZ, Gilette JR, Brodie BB 1973 Acetaminophen induced hepatic necrosis. IV. Protective role of glutathione. J Pharmacol Exp Ther 187:211

22. Nakao K, Wada O, Kitamura T, Uono K, Urata G 1966 Activity of $\Delta$ aminolevulinic acid synthetase in normal and porphyric human livers. Nature 210:838

23. Novi Am 1981 Regression of aflatoxin $B_{1}$ induced hepatocellular carcinomas by reduced glutathione. Science 212:541

24. Prescott LF, Park J, Ballantyne A, Andriaenssens P, Proudfoot AT 1977 The treatment of paracetamol (acetaminophen) poisoning with $\mathrm{N}$-acetyl cysteine. Lancet 2:432

25. Rifkind AB, Tseng L, Hirsch MB, Lauersen NH 1978 Aryl hydrocarbon hydroxylase activity and microsomal cytochrome content of human fetal tissues. Cancer Res 38:1572

26. Rifkind AB, Troeger M, Petschke T 1979 Equality of the rates of mixed function oxidations in the livers of male and female chick embryos. Biochem Pharmacol 28:1681

27. Rifkind $\mathrm{AB}$, Petschke T 1981 P-Chloro- $N$-methylaniline-demethylase activity in liver and extrahepatic tissue of the human fetus and in chick embryo liver. J Pharmacol Exp Ther 217:572

28. Rosin WP, Stitch HF 1978 Inhibitory effect of reducing agents on $\mathrm{N}$-acetoxy and $N$-hydroxy-2-acetylaminofluorene induced mutagenesis. Cancer Res $38: 1307$ 
29. Sassa S, Granick S, Bickers DR, Levere RD, Kappas A 1973 Studies on the inheritance of human erythrocyte $\Delta$-aminolevulinic dehydratase and uroporphyrinogen synthetase. Enzyme 16:325

30. Sassa S, Granick JL, Granick S, Kappas A, Levere RD 1973 Studies in lead poisoning. I. Microanalysis of erythrocyte protoporphyrin levels by spectrofluorometry in the detection of chronic lead intoxification in the subclinical range. Biochem Med 8:135

31. Sassa S, Kappas A, Bernstein SE, Alvares AP 1979 Heme biosynthesis and drug metabolism in mice with hereditary hemolytic anemia. $\mathrm{J}$ Biol Chem 254:729

32. Strife CG, Zuroweste EI, Emmett EA, Finelli VN, Petering HC, Berry HK 1977 Tyrosinemia with acute intermittent porphyria: $\Delta$-aminolevulinic acid dehydratase deficiency related to elevated urinary aminolevulinic acid levels. J Pediatr 90:400

33. Tietze F 1969 Enzymic method for quantitative determination of nanogram amounts of total and oxidized glutathione. Anal Chem 27.502

34. Tschudy DP, Perlroth MG, Marver HS, Collins A, Hunter G Jr, Rechcigl M Jr 1965 Acute intermittent porphyria: the first "overproduction disease" localized to a specific enzyme. Proc Natl Acad Sci USA 53:841

35. Weinberg AG, Mize CE, Worthen HG 1976 The occurrence of hepatoma in the chronic form of hereditary tyrosinemia. J Pediatr 88:434

36. Whelan DT, Zannoni VG 1974 Microassay of tyrosine amino transferase and p-hydroxyphenylpyruvic oxidase in mammalian liver and patients with hereditary tyrosinemia. Biochem Med 9:19

37. Williamson JM, Meister A 1981 Stimulation of hepatic glutathione formation by administration of L-2-oxothiazolidine-4-carboxylate, a 5-oxo-L-prolinase substrate. Proc Natl Acad Sci USA 78:936

38. Williamson JM, Boettcher B, Meister A 1982 Intracellular cysteine delivery system that protects against toxicity by promoting glutathione synthesis. Proc Natl Acad Sci USA 79:6246

\title{
$\mathrm{Cl}^{-}$Permeabilities in Red Blood Cells and Peripheral Blood Lymphocytes from Cystic Fibrosis and Control Subjects
}

\author{
RICHARD C. BOUCHER, DENNIS W. ROSS, MICHAEL R. KNOWLES, JOHN T. GATZY, AND \\ JOHN C. PARKER \\ Departments of Medicine, Pathology, and Pharmacology, University of North Carolina at Chapel Hill, \\ North Carolina 27514
}

\begin{abstract}
Recent studies have identified abnormalities in $\mathrm{Cl}^{-}$permeation across two target cystic fibrosis (CF) epithelia (sweat duct and respiratory epithelium). In the present study, anion conductances of red blood cells (RBCs) and peripheral blood lymphocytes (PBLs) from CF and normal subjects were estimated and compared. For RBCs, the valinomycin-induced rate constant for $K^{+}$loss $\left(P_{K^{+}}\right)$ was taken as an index of $\boldsymbol{P}_{\mathrm{Cl}^{-}}$. For PBLs, the secondary volume increase after gramicidin pretreatment and hypotonic $\left(0.67 \times\right.$ isotonic) stress was used to estimate $P_{\mathrm{Cr}}$. The $\mathrm{Cl}^{-}$permeabilities of RBCs and PBLs from CF and control subjects were comparable. These findings suggest that the abnormality in $\boldsymbol{P}_{\mathrm{Cl}^{-}}$reported for $\mathrm{CF}$ sweat ductal and respiratory epithelia is not expressed in circulating blood elements. (Pediatr Res 18:1336-1339, 1984)
\end{abstract}

\section{Abbreviations}

$\mathrm{CF}$, cystic fibrosis

PBL, peripheral blood lymphocyte

RBC, red blood cell

$P_{\mathrm{Cl}^{-}}, \mathrm{Cl}^{-}$permeability coefficient

$P_{\mathrm{K}^{+}}, \mathrm{K}^{+}$permeability coefficient

HEPES, 4-(2-hydroxyethyl)-1-piperazineethanesulfonic acid

Received January 2, 1984: accepted April 27, 1984.

Please direct correspondence to Richard C. Boucher, M.D., Department of Medicine. 742 Burnett-Womack Bldg. 229H, University of North Carolina, Chapel Hill. NC 27514.

This work was supported by HL 22924, HL 16674; HL 00787, and AM 11357 , the University of North Carolina Blood Cell Fund, and by a Cystic Fibrosis Foundation Research Resources Development Program. R.C.B. is an Established Investigator of the American Heart Association.

\author{
DIDS, 4-acetamide-4'-isothiocyanostilbene-2,2' -disul- \\ fonic acid \\ SITS, 4,4'-diisothiocyanostilbene-2,2'-disulfonic acid
}

Cystic fibrosis is a disease that is characterized by multiple abnormalities in the volume and composition of body "secretions" (5). This feature has led many investigators to suggest that the disease may reflect a generalized dysfunction in ion permeation (5). Evidence has accrued that the CF sweat ductal epithelium may be relatively impermeable to $\mathrm{Cl}^{-}$ions $(23,26)$. A reduction in cell $\mathrm{Cl}^{-}$permeability also appears to be a characteristic of CF respiratory epithelia $(17,18)$. The defect at each site appears to involve a path for $\mathrm{Cl}^{-}$that is electrically conductive. It is not yet known whether this dysfunction reflects a structural or functional (control) abnormality.

Techniques have recently been developed to identify and partition $\mathrm{Cl}^{-}$movements across membranes of circulating blood elements to either conductive or nonconductive paths. In the $\mathrm{RBC}$, ion and water movement across valinomycin-treated cells reflect conductive $\mathrm{Cl}^{-}$flows $(13,14)$. In the PBL, volume regulatory changes in response to an isosmotic medium have been used to study conductive paths for $\mathrm{K}^{+}$and $\mathrm{Cl}^{-}(9,10)$. Because an abnormality in $\mathrm{Cl}^{-}$channels might be generalized to blood elements in CF subjects, we compared the magnitude and regulation of conductive $\mathrm{Cl}^{-}$paths in RBCs and PBLs from $\mathrm{CF}$ subjects with those from control (normal) subjects.

\section{MATERIALS AND METHODS}

Subjects. Blood was obtained in heparinized tubes and used for study within $4 \mathrm{~h}$ of venipuncture. Blood was obtained from 